\title{
Post-Stall Aerodynamic Modeling and Gain-Scheduled Control Design
}

\author{
Fen $\mathrm{Wu}^{*}$ and Ashok Gopalarathnam ${ }^{\dagger}$ \\ North Carolina State University, Raleigh, NC 27695 \\ Sungwan Kim Kim $^{\ddagger}$ \\ NASA Langley Research Center, Hampton, VA 23681-2199
}

\begin{abstract}
A multidisciplinary research effort that combines aerodynamic modeling and gain-scheduled control design for aircraft flight at post-stall conditions is described. The aerodynamic modeling uses a decambering approach for rapid prediction of post-stall aerodynamic characteristics of multiple-wing configurations using known section data. The approach is successful in bringing to light multiple solutions at post-stall angles of attack right during the iteration process. The predictions agree fairly well with experimental results from wind tunnel tests. The control research was focused on actuator saturation and flight transition between low and high angles of attack regions for near- and post-stall aircraft using advanced LPV control techniques. The new control approaches maintain adequate control capability to handle high angle of attack aircraft control with stability and performance guarantee.
\end{abstract}

\section{Introduction}

$\mathrm{T}$ HE potential for significant benefits from controlled flight of aircraft at near-stall and post-stall conditions makes it an attractive area for research. The aim of this multidisciplinary research project is to advance the state of the art in aerodynamic characterization and flight control of aircraft at high post-stall angles of attack. The potential benefits include reduction in landing distances, capability to make emergency evasive maneuvers to avoid collisions and avoid controlled flight into terrain, and maintaining control in the presence of severe atmospheric turbulence.

In order to design effective controllers for such flight regimes, it is necessary to get reliable, yet rapid aerodynamic modeling techniques that can predict the post-stall aerodynamics of full aircraft (with multiple lifting surfaces). Thus, a major part of this research effort aims at developing methods for rapid, albeit approximate, prediction of post-stall wing aerodynamic characteristics. For this objective we have developed a decambering approach for multiple lifting surfaces that uses known two-dimensional airfoil characteristics as input. Earlier approaches for prediction of wing aerodynamics using nonlinear airfoil lift curves can be broadly classified into two kinds: (a) iterative $\Gamma$-distribution approaches ${ }^{1-8}$ and (b) $\alpha$-reduction approaches. ${ }^{9,10}$ The current approach is similar to the $\alpha$-reduction approach, but uses a novel scheme for the residual computation for the Newton iteration. This approach and results from the current method are presented in Sec. II.

\footnotetext{
*Corresponding author, Associate Professor, Dept. of Mechanical and Aerospace Engineering. Member AIAA. E-mail: fwu@eos.ncsu.edu, Phone: (919) 515-5268, Fax: (919) 515-7968.

$\dagger$ Associate Professor, Dept. of Mechanical and Aerospace Engineering. Senior Member AIAA. E-mail: ashok_g@ncsu.edu

$\ddagger$ Research Engineer, Dynamic Systems and Control Branch. Associate Fellow AIAA. E-mail: s.kim@larc.nasa.gov
} 
Because aerodynamic controls may be insufficient for control at post-stall conditions, the aircraft models including thrust vectoring (TV) control effectors have been developed to augment the conventional controls. Robust multivariable control methods have been recently applied to a variety of aircraft models to demonstrate their ability to fly at high angles of attack with the help of thrust vectoring control (see Ref. 11 and references therein). As an alternative, an antiwindup compensation scheme was first studied for aircraft actuator saturation in a high angle of attack region without TV effectors. The research was then focused on the development of a robust, gain-scheduling control system that can not only handle the uncertainties in the environment and the aerodynamic and TV models, but also allow for adjustment of the controller gains based on the operating condition. The gain-scheduled control designs smoothly transition between aerodynamic controls and TV controls. In addition, the designed controllers also provide explicit stability and performance guarantees for a large flight envelope, including post-stall region. The design approaches and simulation results are presented in Section 3.

\section{Modeling of Post-Stall Aerodynamics}

In this section, a novel scheme, based on an iterative decambering approach, is presented for the prediction of post-stall characteristics of wings using known section data as inputs. The scheme is suitable for implementation in lifting-line and vortex-lattice methods. The following subsections illustrate the decambering approach, provide an overview of the methodology, and present example results from the method.

\section{A. Illustration of the Decambering Concept}

With increasing angle of attack, the boundary layer on the upper surface of an airfoil thickens and finally separates. It is this flow separation that causes the viscous $C_{l}$ and $C_{m}$ to deviate from the potential-flow theory predictions. These deviations can be related to the effective change in the airfoil camber distribution due to the boundary-layer displacement thickness and separation. If the effective decambering is taken into account, then a potential-flow prediction for the decambered airfoil will closely match the viscous $C_{l}$ and $C_{m}$ for the high- $\alpha$ flow past the original airfoil shape. This decambering idea served as the basis for the formulation of the current approach for the three-dimensional flow problem.

In the current method, the effective decambering of an airfoil is approximated using a function of two variables, $\delta_{1}$ and $\delta_{2}$, as shown in Fig. 1. These two linear functions are added to obtain the final decambering function. The reason for using two variables is that the decambering is determined from two pieces of information: the $C_{l}$ and the $C_{m}$ for the $\alpha$ under consideration. Of course, this approximation will not match the actual viscous decambering, but the objective here is only to find an equivalent camber reduction in order to match the viscous $C_{l}$ and $C_{m}$ for the $\alpha$ under consideration. For the details of how the two variables, $\delta_{1}$ and $\delta_{2}$, are determined from the $C_{l}$ and $C_{m}$ for any given $\alpha$ and a demonstration of the effectiveness of the decambering concept, the reader is referred to Ref. 12 .

\section{B. Methodology}

The objective of the research was to incorporate the two-variable decambering function in a three-dimensional analysis method such as a vortex lattice method (VLM) in an iterative fashion. In a typical VLM, the lifting surface is divided into several spanwise and chordwise lattices. Associated with each lattice is a horseshoe vortex. Each spanwise section $j$ (composed of a row of chordwise lattices) has two variables, $\delta_{1 j}$ and $\delta_{2 j}$, for defining the local decambered geometry at that section. A Newton iteration is used to predict the $\delta_{1}$ and $\delta_{2}$ at each section so that the $\Delta C_{l}$ and $\Delta C_{m}$ at these sections approach zero as the iteration progresses.

In the current research, two schemes were developed for the Newton iteration. The two schemes differ in the details of how the residuals are computed. The first scheme, introduced in Ref. 15, was found to work well for certain airfoil lift curves, but failed to converge for several other lift-curve shapes. This lack of robustness provided the impetus for developing the second scheme. These two schemes have been incorporated in a 
custom vortex lattice method, VLM3D, and in a discrete-vortex Weissinger's method code, WINGS. All the results in this paper are from the VLM3D code. For the additional details of the iteration scheme, the reader is referred to Ref. 12. In this paper, only the main difference between the two schemes will be discussed.

The two schemes are described using the illustration in Fig. 2(a). We consider a section of the wing at some intermediate step in the Newton iteration. Let $\delta$ be the starting value of a decambering variable for this step of the iteration, and let the operating local lift coefficient of this section be $C_{l s}$ and operating effective angle of attack be $\alpha_{s}$, where $s$ denotes the start of the iteration. The residual for the Newton iteration is simply the $\Delta C_{l}$, which is $C_{l s}-C_{l t}$, where $C_{l t}$ is the target $C_{l}$. In scheme 1 , the target $C_{l}$, denoted by $C_{l t, 1}$, is chosen as the $C_{l}$ on the airfoil lift curve corresponding to $\alpha_{s}$, as shown in Fig. 2(a). This formulation is similar to those used in other $\alpha$-correction methods. ${ }^{9,10}$

The formulation for scheme 2, on the other hand, takes into consideration that when the decambering at a section is changed, there is a change not only to the operating $C_{l}$, but also to the operating effective angle of attack of the section. As illustrated in Fig. 2(a), when the decambering is perturbed from $\delta$ to $\delta+p$, the perturbed operating condition for the section becomes $C_{l p}$ and $\alpha_{p}$. This perturbed operating condition can be determined using the VLM. The line joining the points $\left[\alpha_{s}, C_{l s}\right]$ and $\left[\alpha_{p}, C_{l p}\right]$ for any section is called the "trajectory line" for that section, as it determines the linearized trajectory of how a point on the $C_{l^{-}} \alpha$ curve defined by the section $\alpha_{e f f}$ and section $C_{l}$ moves with changes to the decambering for that section. This trajectory line is illustrated in Fig. 2(a). Therefore, in scheme 2, the target $C_{l},\left(C_{l t, 2}\right)$ for the section is the point of intersection between the trajectory line and the airfoil lift curve for the section as illustrated in Fig. 2(a).

Figure 2(b) illustrates an important consequence of using an inclined trajectory line for determining the target $C_{l}$ in scheme 2 . Three possible ways in which the trajectory line may intersect the airfoil lift curve are illustrated in Fig. 2(b): (i) the trajectory line marked as L1 intersects the airfoil lift curve at a single pre-stall point, (ii) the trajectory line marked as L2 intersects the airfoil lift curve at multiple points and (iii) the trajectory line marked as L3 intersects the airfoil lift curve at a single point in the post-stall region. While there is no ambiguity in determining the values of the target $C_{l}$ for lines L1 and L3, there clearly are three possible choices for the target $C_{l}$ for line L2. This illustration clearly demonstrates that it is possible to obtain multiple solutions for post-stall conditions; a fact, that was apparently first suggested by von Kármán (see Ref. 4) and has since been discussed by several researchers. ${ }^{3-8,15}$ However, the approach in scheme 2 is novel because this scheme is believed to be the first one in which the possibility of multiple solutions for high angles of attack is brought to light right during the iteration process. Earlier approaches including scheme 1 were able to identify the existence of multiple solutions only as a result of obtaining multiple final converged solutions with different initial conditions for the iteration procedure.

The existence of multiple intersections also presents a dilemma in choosing an appropriate target $C_{l}$ from the possible multiple solutions. The following procedure was developed for making the choice during the iteration process. At each step of the iteration, each section is examined to identify those with single intersections, as identified by points $\mathrm{A}$ and $\mathrm{B}$ in Fig. 2(b). The target $C_{l}$ values for these sections are identified without ambiguity. Using a logical switch called "lpoststall" in the code, each section is also tagged as "stalled" or "unstalled" depending on whether the $\alpha$ for the intersection point is greater than or less than the $\alpha$ for $C_{l \max }$. For example, lpoststall is tagged unstalled for point A and stalled for point B in Fig. 2(b). The sections with multiple intersections are then examined. Using the trajectory line L2 in Fig. 2(b) for example, the intersection point 1 is chosen if the logical switch lpoststall for the section is unstalled and the intersection point 3 is chosen if the logical switch for the section is stalled.

Next, another logic is applied in which all the sections of the wings are scanned to identify sets of contiguous sections, all of which have multiple intersections and all of which are also tagged as unstalled. If any of these sets of contiguous sections are bound on both sides by sections tagged as stalled, then all the sections in this set are switched to stalled. This logic largely removed any occurrence of unstalled regions with multiple intersections sandwiched between two stalled regions. 


\section{Results}

Two examples are presented to illustrate the decambering approach. The first example provides comparison with experimental data. The second example shows how the method can be applied to multiple-lifting-surface configurations. For further examples, the reader is referred to Ref. 12.

\section{Example 1: Experimental Validation}

In this example, experimental two-dimensional data ${ }^{16}$ for an NACA 4415 airfoil at a Reynolds number of 0.5 million, shown in Fig. 3(a), is used as input to the VLM3D code to generate post-stall results for an unswept rectangular wing of aspect ratio 12. The computational results for the wing of aspect ratio 12 are then compared with the corresponding experimental $C_{L^{-}} \alpha$ curve from Ref. 16. Figure 3(b) shows the wing $C_{L^{-}} \alpha$ curves from VLM3D using schemes 1 and 2 . In the same figure, the airfoil $C_{l^{-}} \alpha$ curve and the wing $C_{L^{-}} \alpha$ curve from experiment ${ }^{16}$ are also shown for comparison.

As seen from Fig. 3(b), the predictions for the values of wing $C_{L}$ at pre-stall angles of attack from the two schemes are identical. For post-stall conditions, the results of scheme 2 are closer than those of scheme 1 to the experimental results. Figures 4(a) and 4(b) show the spanwise $C_{l}$ distributions from the current method for angles of attack 18,21,32, and 36 deg from schemes 1 and 2 respectively. For all of the results presented in Figs. 4(a) and 4(b), convergence was achieved to within a tolerance of 0.001 in $\Delta C_{l}$ and $\Delta C_{m}$. Experimental results for the spanwise $C_{l}$ distributions were not available for comparison. As seen from the results of scheme 1 in Fig. 4(a), there is substantial "sawtooth oscillation" in the spanwise $C_{l}$ distributions with regions of unstalled flow sandwiched between regions of stalled flow. Such undesirable spanwise sawtooth oscillations are a common problem in numerical techniques that use known section data for the prediction of wing post-stall characteristics using, as noted by other researchers. ${ }^{6-8}$ Although these sawtooth results correspond to numerically converged solutions, it is believed that a real flow cannot have such spanwise oscillations. The results from scheme 2, shown in Fig. 4(b), do not exhibit these sawtooth oscillations for a majority of the conditions. For this reason, scheme 2 is considered more acceptable. Further discussion of the sawtooth behavior and its cause is provided in Ref. 12

Example 2: Wing-Tail Configuration

This example illustrates the effectiveness of the method for a multiple-lifting-surface configuration. In this example, results are presented for a high aspect ratio $(\mathrm{AR}=10)$ constant-chord wing geometry with a conventional aft tail. The planform for the wing-tail configuration is shown in Fig. 5(a). For this example, a single airfoil is assumed for both the wing and tail.

Figure 5(b) shows the predicted $C_{L}-\alpha$ curve for the wing-tail configuration with scheme 2 . In the same figure, the airfoil $C_{l^{-}} \alpha$ curve used for this example is included for comparison. Figure 5(b) also shows the $C_{L, w}$ and $C_{L, t}$ as a function of $\alpha$. The $C_{L, t}$ in this plot is nondimensionalized with reference to the tail area. It can be seen that the wing stalls when $\alpha$ just exceeds $17 \mathrm{deg}$. In this example, the tail remains unstalled over the entire $\alpha$ range considered. The spanwise $C_{l}$ distributions are shown in Fig. Fig. 6(a) for $\alpha$ values of 10, 15, 18, and $23 \mathrm{deg}$. These values were chosen for illustrating the stall behavior.

Of particular interest when studying wing-tail configurations is the aircraft pitching moment about the center of gravity (CG). For this analysis, the CG was located to provide a static margin of $10 \%$ of the wing mean aerodynamic chord. A tail incidence of $-5 \mathrm{deg}$ was chosen and results in the aircraft being trimmed at $\alpha$ close to $13 \mathrm{deg}$. In other words, the $C_{M_{c g}}$ is nearly zero at $\alpha$ of $13 \mathrm{deg}$. Figure $6(\mathrm{~b})$ shows the variation of the aircraft $C_{M c g}$ as a function of $\alpha$. As the $\alpha$ is increased, a distinct increase in the nose-down pitching moment coefficient (negative $C_{M c g}$ ) is seen at the onset of stall at $\alpha$ of $17 \mathrm{deg}$.

To examine the cause of this nose-down pitching moment at stall onset, it is instructive to study the individual contributions of the wing and the tail to the configuration lift curve shown in Fig. 5(b). It can be seen that there is a noticeable increase in the tail $C_{L, t}$ at the onset of wing stall at $\alpha$ of $17 \mathrm{deg}$. This increase in $C_{L, t}$ at stall onset contributes significantly to the increase in the nose-down pitching moment at stall. 
The origin of the sudden increase in $C_{L, t}$ at stall can in turn be traced to the shape of the wing spanwise lift distribution at the onset of stall. As seen earlier in Fig. 6(a), the wing stall occurs at the root for $\alpha$ of $18 \mathrm{deg}$ for this example. This loss in lift over the inboard portion of the wing results in a reduction in the downwash at the tail. In addition, it also results in a pair of trailing vortices originating from the outboard ends of the stalled portion of the wing (at $y /(b / 2)$ of approximately \pm 0.25$)$. These trailing vortices cause an upwash at the tail. As a consequence of these two factors, there is a distinct increase in the $C_{L, t}$ and a resulting increase in the nose-down pitching moment at the onset of stall.

Thus, as illustrated by this simple example, a method that can predict the post-stall characteristics of wing-tail configurations can provide important information for the study of aircraft longitudinal behavior at these post-stall conditions. These results can be further improved by the addition of models for taking into consideration the wake deformation and the dynamic-pressure loss in the wing wake.

\section{LPV Control for Post-Stall Flight}

\section{A. Antiwindup control to alleviate saturation effect}

High angle of attack flight presents many challenges to the control designers. Saturation control is particularly important to near stall and post-stall flight conditions. Due to aerodynamic surface saturation and control surface limitation, unconventional actuators such as thrust vectoring were suggested for aircraft maneuvering at and beyond the stall angle of attack.

In this research, a saturation control scheme was developed for linear parameter-varying (LPV) systems from an antiwindup control perspective. ${ }^{17,19}$ The proposed control approach is advantageous because it can be thought of as an augmented control algorithm from the existing control system. Due to the special structure of the antiwindup control scheme, the LPV antiwindup control synthesis condition is solvable by LMI optimizations efficiently ${ }^{19}$ (see Fig. 7). The LPV antiwindup controller has been applied to an F-16 longitudinal autopilot control system design to enhance aircraft safety and improve flight quality in a high angle of attack region. By augmenting the nominal longitudinal autopilot with an antiwindup compensator, it was shown through nonlinear simulation that the F-16 aircraft maintains its stability and has adequate control performance. Applying antiwindup control scheme to flight control is promising because no additional actuator is needed to implement antiwindup compensator. In fact, the implementation of antiwindup controllers could be done by modifying flight control software.

To describe the nonlinear F-16 model by an LPV system, the wings-level equilibrium solutions at multiple selected flight conditions in the design envelope needs to be determined. The local linear models are then obtained by linearizing the nonlinear equations of motion at those equilibrium points. The flight envelope of interest covers aircraft speeds between 160 and $200 \mathrm{ft} / \mathrm{s}$ and angles of attack between 20 and $45 \mathrm{deg}$. These two variables are used as scheduling parameters in the LPV modeling of F-16 longitudinal dynamics. The points at which the nonlinear model is linearized are marked by a $\times$ symbol in Fig. 8. This group of linearized models consist of the LPV representation of the nonlinear F-16 longitudinal dynamics within the chosen flight envelope. The corresponding dynamic pressure range is between 20 and $50 \mathrm{lb} / \mathrm{ft}^{2}$. This portion of the flight envelope is chosen because the moderately high angle-of-attack and the low dynamic pressure cause aerodynamic control surface saturation, which is a major concern in this research. The actuators are explicitly constrained. Most of the time, the elevator angle saturation was found to be a limiting factor for flight control effectiveness.

Note that the nominal controller $K_{\rho}$ is designed for a set of linearized plant models at the gridding points, so that the output of $K_{\rho}$ is the control deviation from the nominal control maintaining trim condition, and the actual control input to the nonlinear plant should be the summation of both terms. When $K_{\rho}$ is parameter varying in simulations, the instant nominal control inputs should be determined at each time. This can be done by building up another look-up table of nominal control inputs at finer gridding points beforehand and interpolating in real time according to the current parameter values.

For the flight condition just listed, a flight-path doublet input with magnitude $\pm 4 d e g$ is used for simulation

5 of 15

American Institute of Aeronautics and Astronautics 
purpose. The simulation results have confirmed that the trajectory of parameters involves about half of the parameter space. In this case, the system might not achieve the desired performance, even maintain stability by using a single LTI antiwindup compensator. Instead, it is crucial to use LPV nominal control and LPV antiwindup compensation to achieve good controlled performance. Fig. 9 shows the aircraft response with an LPV antiwindup compensator, and the dotted line in Fig. 9(a) is the ideal flight-path angle response. It is observed that the LPV antiwindup compensator achieves the desired performance objective. However, when the antiwindup compensator is unused, both the elevator and the throttle are saturated severely and the system goes unstable. For clarity purpose, the response without LPV antiwindup compensator is not shown in Fig. 9.

\section{B. Switching control for performance trade-off}

On the other hand, different performance goals are often desirable for different angle of attack regions in flight control. For example, in a low angle of attack scenario, pilots desire fast and accurate responses for maneuvering and attitude tracking. While in a high angle of attack region, the flight control emphasis lies in the maintainability of aircraft stability with acceptable flying qualities. A modern fighter aircraft usually works in a wide angle of attack region, even a near stall or post-stall regime. In such a case, it is difficult to design a single robust controller over the entire flight envelope. Typically, the controller was designed by compromising the performance in some angle of attack region.

To avoid those problems, a systematic switching linear parameter-varying (LPV) control technique was proposed to design a family of controllers, each suitable in different angle of attack regions, and switch among them according to the evolution of angle of attack. ${ }^{18}$ The switching LPV control approach is based on multiple parameter-dependent Lyapunov functions, and is very useful for highly maneuverable aircraft control design. The state of the controller is reset to the value of the plant state when the switching event occurs (assuming plant state information is available at switching instance). The controller state reset not only guarantees the stability requirement of Lyapunov function, but also leads to the formulation of switching LPV control synthesis conditions as LMI optimization problems. Two parameter-dependent switching logics, hysteresis-based switching and switching with average dwell time, are used to avoid the possible transient instability caused by switching among controllers. The proposed switching LPV control technique is applied to an F-16 aircraft model switching between low and high angle of attack regions with different control objectives and actuator sets, and promising simulation results are obtained. ${ }^{20,21}$

The flight envelope of interest covers aircraft speeds between $160 \mathrm{ft} / \mathrm{s}$ and $200 \mathrm{ft} / \mathrm{s}$ and angles of attack from $20^{\circ}$ to $45^{\circ}$. These two variables are used as scheduling parameters in the LPV modeling of F-16 longitudinal dynamics. The points at which the nonlinear model is linearized are marked by an " $\times$ " symbol in Fig. 10. To apply the switching LPV control synthesis technique, the flight envelope is partitioned into two subregions. For hysteresis-based switching, the striped area in Fig. 8(a) is the overlapped parameter region. So there are two switching surfaces: $\alpha=30^{\circ}$ and $\alpha=35^{\circ}$. For the switching with average dwell time, the overlapped region shrinks to a line. The switching surface $\alpha=33^{\circ}$ has been shown in Fig. 8(b).

In this research, two different sets of actuators are used in the different angle of attack regions. The actuators used in the low angle of attack region (region 1) are the throttle and the elevator, and the thrust vectoring nozzle is inactive. The local linear models in this region are based on the original aircraft model, which corresponds to the case of $\delta_{p t v}=0$. In the high angle of attack region (region 2), the thrust vectoring nozzle is incorporated to provide additional force and moment. Therefore, the switching of controllers is based on the trajectory of the angle of attack, that is, the controller is switched only when the aircraft flies from one angle of attack region to the other.

To test the performance of the switching control system during the nonlinear simulation, two angle of attack command inputs are defined in Fig. 11. The initial angle of attack command is selected as $36^{\circ}$, and thus the initial switching signal $\pi(0)=2$. The trajectory of the angle of attack command is chosen as square waves and deliberately cross the two parameter subsets back and forth to illustrate the effect of switching LPV control. There are four switching events happening. For the first command, the switches occur at $0 s$, 
$15 s, 31 s$, and $45 s$, respectively. Therefore, all the time intervals between switches satisfy the requirement $\tau_{a}>9.53 \mathrm{~s}$ using the dwell time formula in Ref. 21. To see the effect of the average dwell time $\tau_{a}$, we defined another command input that switches fast at the beginning and then compensates by switching sufficiently slow. The switches occur at $0 s, 5 s, 10 s$, and $45 s$, respectively, and thus the average dwell time still satisfy the requirement. Note that the switching time of the actual angle of attack trajectory is slightly different from the command input because the aircraft tries to track the response of a second-order ideal model, not the square wave.

The test flight condition is selected at $V=180 \mathrm{ft} / \mathrm{s}$ and $\alpha=36^{\circ}$. Fig. 12 shows the nonlinear responses of the aircraft model for the command input 1. The dotted lines in subplot (a) represent the angle of attack response of the ideal model, the solid and dashed lines in all subplots represent the responses using hysteresis switching and average dwell time switching, respectively. The tracking performances over the entire time history are acceptable for both switching logics. Note that the switching signal $\pi$ in subplot (b) is a little different for those two switching logics because their switching surfaces are different. For hysteresis switching, the switch event occurs when $\alpha=30^{\circ}$ or $35^{\circ}$; whereas for average dwell time switching there is only one switching surface $\alpha=33^{\circ}$. The responses of the actuators are shown in subplots (d)-(f). Obviously, the thrust vector is activated only when the aircraft flies in the high angle of attack region.

Figure 13 presents the nonlinear response to command input 2. For the second switching logic, although the first two switch events occur very fast, the average dwell time requirement is satisfied. Therefore, the tracking performance of switching with average dwell time remains acceptable and is a little worse than that achieved by the hysteresis switching. However, the stability of the switched system will not be guaranteed if the condition of the average dwell time is violated. Compared with the average dwell time switching, there is no such restriction on the hysteresis switching, and thus it should be more flexible for the high performance aircraft application.

\section{Conclusions}

Through this research, valuable approaches and tools for aerodynamics modeling and control of flight vehicles in a challenging flight regime have been developed. The research work provides insight into the post-stall flight mechanics and optimized control design strategies which expand the flight envelope and maneuverability of modern aircraft. The methods and tools have the potential for use in developing air vehicles that have post-stall operation capabilities useful for civilian and war fighting airplane applications.

For the prediction of post-stall aerodynamics of wings, a novel iteration scheme has been developed to implement a decambering approach that accounts for the boundary-layer separation effects on each section of the wings of a multiple-lifting-surface configuration. This numerical approach, when incorporated in an analysis method such as a vortex-lattice code, allows for the computation of post-stall aerodynamic characteristics of wings using known section data. While it is recognized that the surface streamlines are no longer two dimensional at post-stall conditions, the current approach aims to provide rapid but approximate solutions for such conditions for use in post-stall flight dynamics and simulation studies and in the early stages of configuration design.

A novel feature of the current approach is that it brings to light the existence of multiple solutions at post-stall conditions right during the iteration process. In contrast, earlier approaches were able to identify the existence of multiple solutions only as a result of obtaining multiple converged solutions with different initial conditions in the iteration procedure. The current scheme, therefore, brings new insight to the iterative solution of wing flows using known airfoil characteristics. The results from the new scheme compare fairly well with wind-tunnel post-stall data for wings and agree with well-known trends in stall patterns. The current approach is shown to have significant potential in providing information for the analysis of aircraft longitudinal stability and control characteristics at post-stall conditions.

An antiwindup control scheme for LPV systems has been developed to compensate actuator saturation. The proposed control approach is advantageous because no additional actuator hardware is needed and can be implemented by modifying flight control software. Due to the special structure of the antiwindup 
control scheme, the LPV antiwindup control synthesis condition is solvable by LMI optimizations. The LPV antiwindup compensation has been applied to an F-16 aircraft successfully. Through nonlinear simulation, it has been shown that the F-16 aircraft maintains stability and adequate control performance in case of actuator saturation.

A switching LPV control approach for large envelope flight control has also been developed using multiple parameter-dependent Lyapunov functions. Two parameter-dependent switching logics, hysteresis-based switching and switching with average dwell time, are used to avoid the possible transient instability caused by switching among controllers. The synthesis problems of both switching controllers have been formulated as LMI optimization problems through controller reset with explicit stability and performance guarantees. The proposed switching LPV control techniques have been applied to an F-16 aircraft model switching between low and high angle of attack regions with different control objectives and actuator sets (aerodynamic surface and thrust vectoring nozzle), and promising simulation results are obtained.

The research has shown significant potential for use in design of aircraft for high angle of attack flight. The research done has established a solid foundation for further exploration of post-stall flight dynamics and control. In follow-on research, the new aerodynamic models will be integrated into the control design process. The aerodynamic models will be expanded to include unsteady effects. The LPV controllers will be generalized to handle six-degree freedom of aircraft dynamics. Simulation results will be used to illustrate the aerodynamic modeling capabilities and predict the performance of the newly designed control system.

\section{Acknowledgment}

The authors would like to acknowledge the contributions of former NCSU Ph.D. students, Rinku Mukherjee and Bei $\mathrm{Lu}$, to this effort.

\section{References}

${ }^{1}$ Tani, I., "A Simple Method of Calculating the Induced Velocity of a Monoplane Wing," Rep. No. 111 (vol. 9, 3), Aero. Res. Inst., Tokyo Imperial Univ., August 1934.

${ }^{2}$ Sivells, J. C. and Neely, R. H., "Method for Calculating Wing Characteristics by Lifting-Line Theory Using Nonlinear Section Lift Data," NACA TN 1269, April 1947.

${ }^{3}$ Schairer, R. S., "Unsymmetrical Lift Distributions on a Stalled Monoplane Wing," Thesis, California Institute of Technology, 1939.

${ }^{4}$ Sears, W. R., "Some Recent Developments in Airfoil Theory," Journal of The Aeronautical Sciences, Vol. 23, May 1956, pp. 490-499.

${ }^{5}$ Piszkin, S. T. and Levinsky, E. S., "Nonlinear Lifting Line Theory for Predicting Stalling Instabilities on Wings of Moderate Aspect Ratio," Tech. rep., General Dynamics Convair Report CASD-NSC-76-001, June 1976.

${ }^{6}$ Levinsky, E. S., "Theory of Wing Span Loading Instabilities Near Stall," AGARD Conference Proceedings No. 204, September 1976

${ }^{7}$ Anderson, J. D., Corda, S., and VanWie, D. M., "Numerical Lifting Line Theory Applied to Drooped Leading-Edge Wings Below and Above Stall" Journal of Aircraft, Vol. 17, No. 12, 1980, pp. 898-904.

${ }^{8}$ McCormick, B. W., "An Iterative Non-Linear Lifting Line Model for Wings with Unsymmetrical Stall," SAE Transactions Paper No. 891020, 1989, pp. 91-98.

${ }^{9}$ Tseng, J. B. and Lan, C. E., "Calculation of Aerodynamic Characteristics of Airplane Configurations at High Angles of Attack," NASA CR 4182, 1988.

${ }^{10}$ van Dam, C. P., Kam, J. C. V., and Paris, J. K., "Design-Oriented High-Lift Methodology for General Aviation and Civil Transport Aircraft," Journal of Aircraft, Vol. 38, No. 6, November-December 2001, pp. 1076-1084.

${ }^{11}$ Adams, R.J., Buffington, J.M., Sparks, A.G., and Banda, S.S., Robust Multivariable Flight Control, Springer-Verlag, London, UK, 1994.

${ }^{12}$ R. Mukherjee, and A. Gopalarathnam, "Post-Stall Prediction of Multiple-Lifting-Surface Configurations Using a Decambering Approach," in Proc. 42nd AIAA Aerospace Sciences Meeting, AIAA Paper 2004-0219, January 2004.

${ }^{13}$ Drela, M., "XFOIL: An Analysis and Design System for Low Reynolds Number Airfoils," Low Reynolds Number Aerodynamics, edited by T. J. Mueller, Vol. 54 of Lecture Notes in Engineering, Springer-Verlag, New York, June 1989, pp. 1-12.

${ }^{14}$ Katz, J. and Plotkin, A., Low-Speed Aerodynamics, Cambridge Aerospace Series, Cambridge University Press, Cambridge, UK, 2nd ed., 2001. 


\begin{abstract}
${ }^{15}$ Mukherjee, R., Gopalarathnam, A., and Kim, S., "An Iterative Decambering Approach for Post-Stall Prediction of Wing Characteristics Using Known Section Data," AIAA Paper 2003-1097, January 2003.

${ }^{16}$ Ostowari, C. and Naik, D., "Post Stall Studies of Untwisted Varying Aspect Ratio Blades with an NACA 4415 Airfoil Section-Part 1," Wind Engineering, Vol. 8, No. 3, 1984, pp. 176-194.

${ }^{17} \mathrm{Wu}$, F. and $\mathrm{Lu}$, B., "Anti-Windup Control Design for Exponentially Unstable LTI Systems with Actuator Saturation," Systems and Control Letters, 52(3/4):305-322, 2003.

${ }^{18}$ F. Wu and Lu, B., "Switching LPV Control Designs using Multiple Parameter-Dependent Lyapunov Functions," Automatica, 40(11):1973-1980, 2004.

${ }^{19} \mathrm{Lu}, \mathrm{B} ., \mathrm{Wu}, \mathrm{F}$. , and Kim, S., "LPV Antiwindup Compensation for Enhanced Flight Control Performance," AIAA Journal of Guidance, Control and Dynamics, 28(3):494-505, 2005.

${ }^{20} \mathrm{Lu}$, B., Wu, F., and Kim, S., "Switching LPV Control of A High Performance Tactical Aircraft," in Proc. AIAA Conference on Guidance, Navigation and Control, AIAA Paper No. 2004-4999, Aug. 2004.

${ }^{21} \mathrm{Lu}, \mathrm{B}$., Wu, F., and Kim, S., "Switching LPV Control of An F-16 Aircraft via Controller State Reset," accepted by IEEE Transactions on Control Systems Technology, June 2005.
\end{abstract}
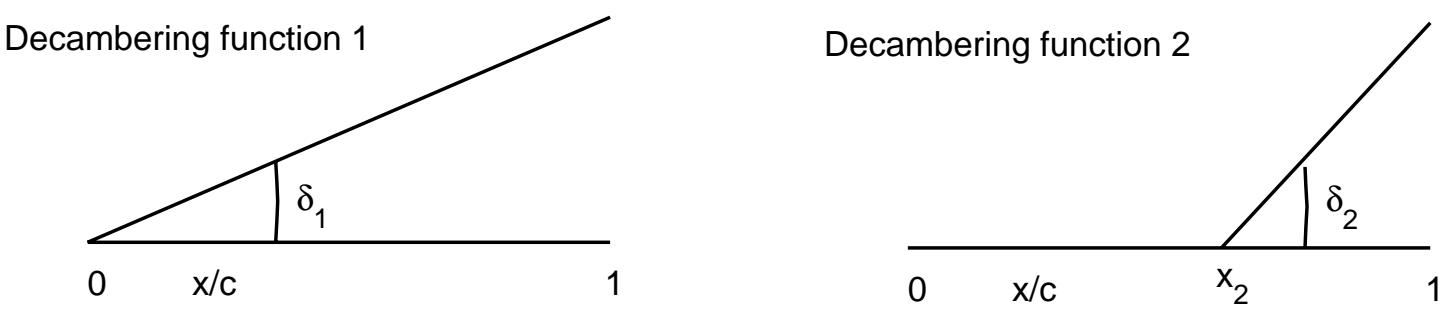

Figure 1. Schematic diagram of decambering functions 1 and $2\left(\delta_{1}\right.$ and $\delta_{2}$ are negative as shown and exaggerated for clarity).

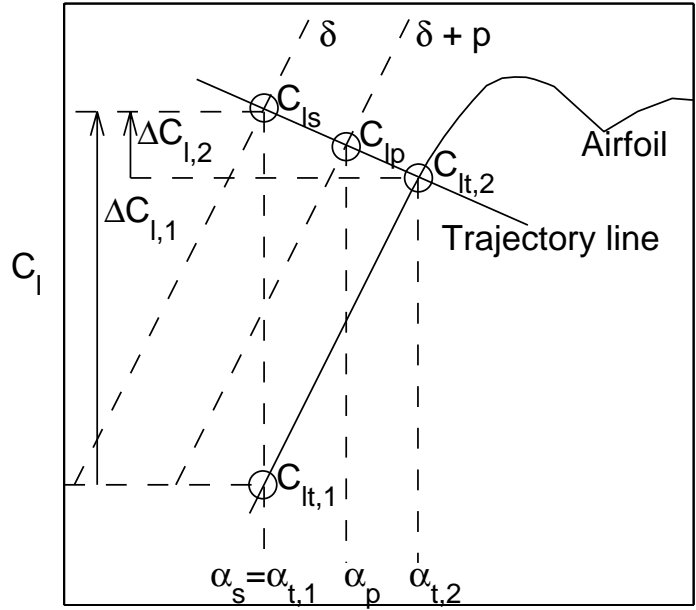

(a) $\alpha$

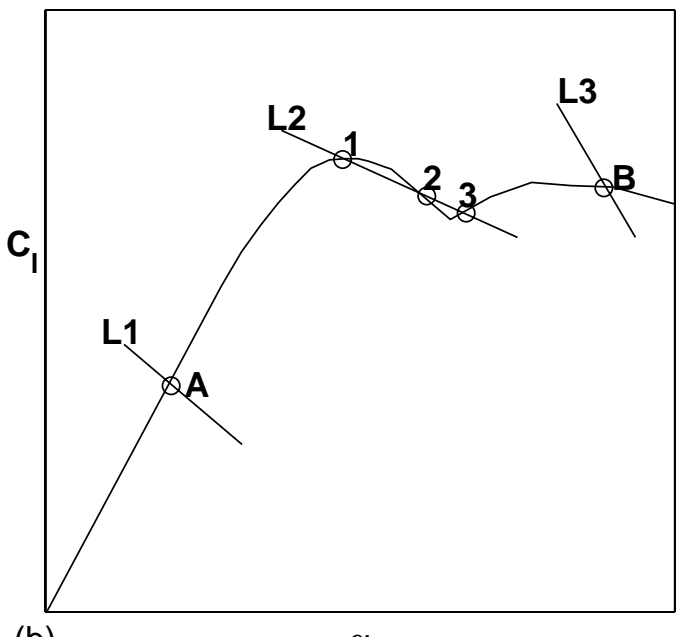

(b) $\alpha$

Figure 2. (a) Illustration of the computation of the residuals for schemes 1 and 2. (b) Illustration of the different ways in which a trajectory line intersects the airfoil $C_{l}$ - $\alpha$ curve. 

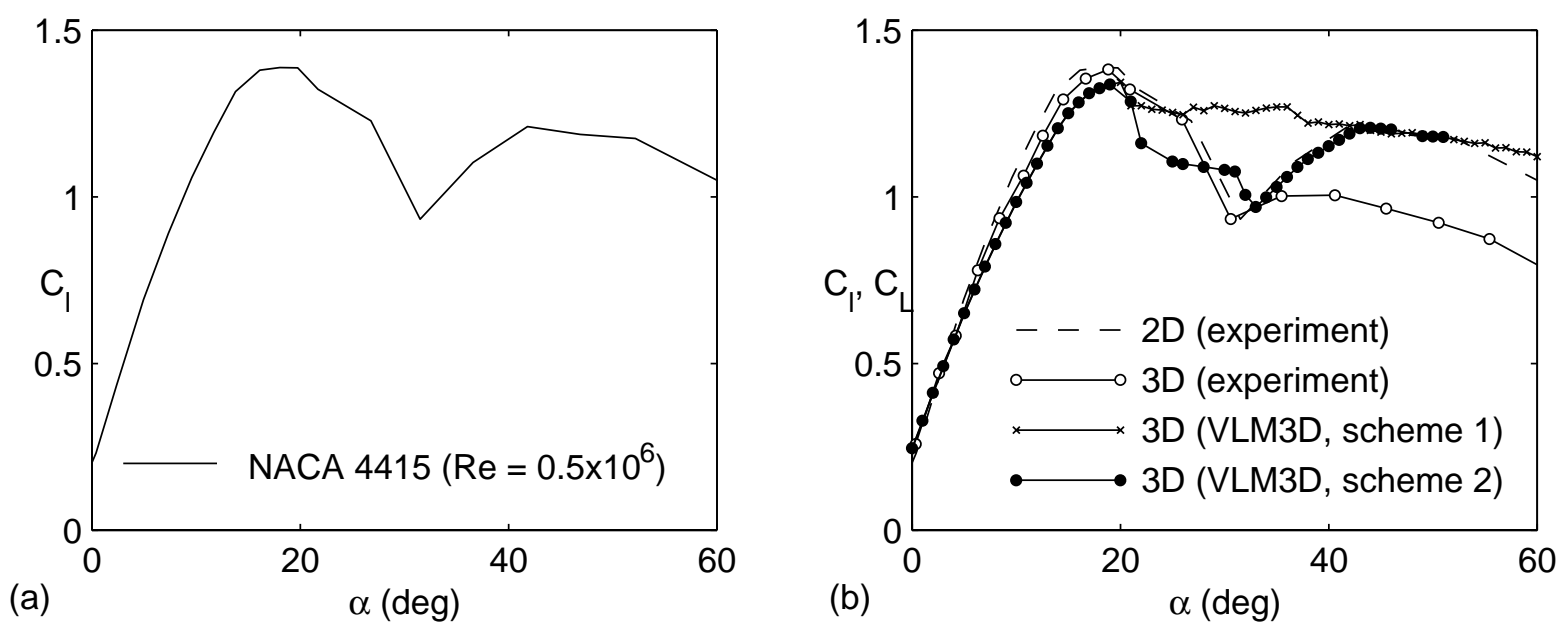

Figure 3. (a) Airfoil $C_{l}$ - $\alpha$ curve used in example 1. (b) Wing $C_{L}-\alpha$ predictions from the two schemes compared with experimental results.
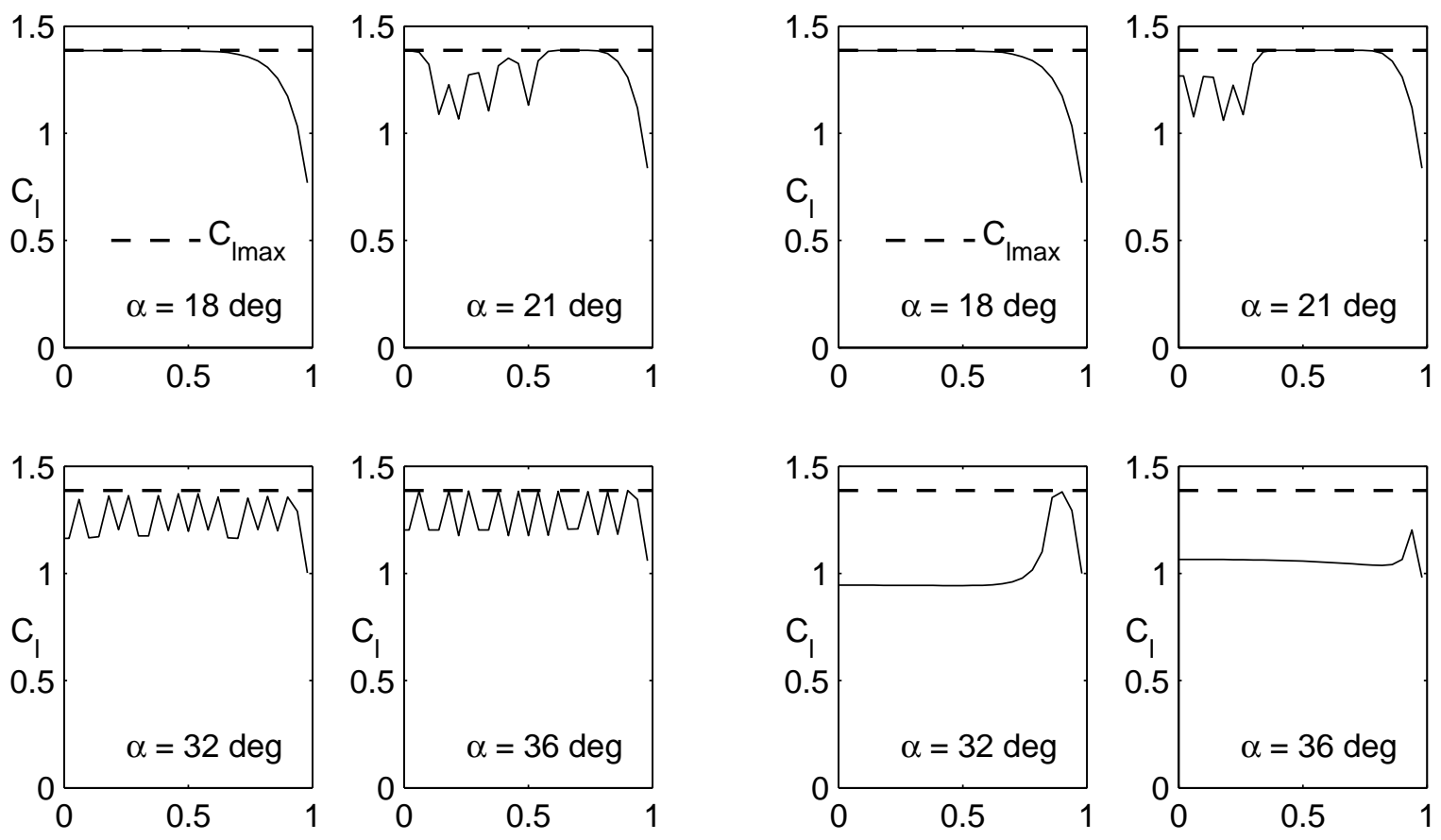

(a) Scheme 1

(b) Scheme 2

Figure 4. Spanwise $C_{l}$ distributions predicted for the rectangular wing of example 1 from the two schemes. 


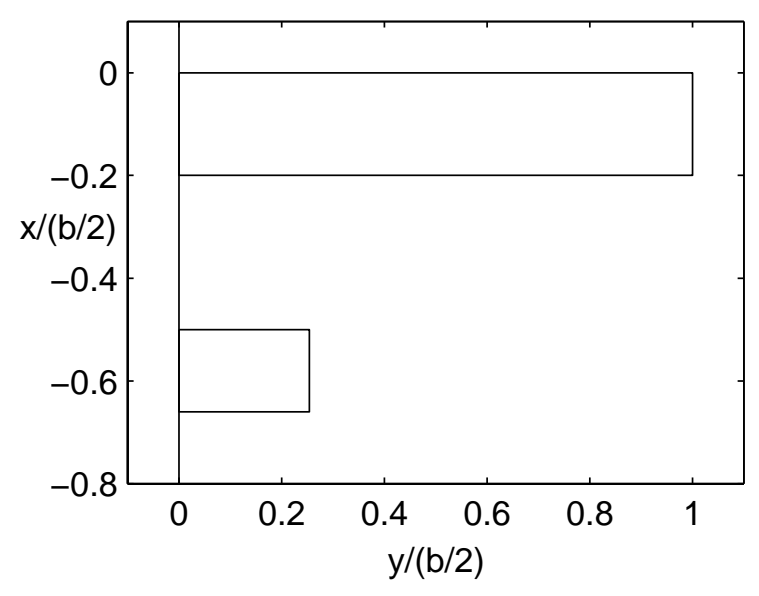

(a) Planform

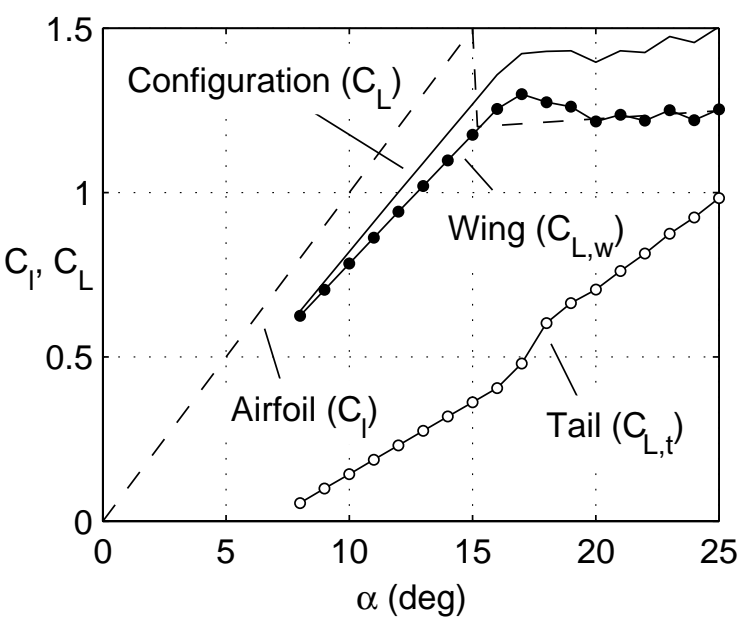

(b) Lift curves

Figure 5. (a) Planform of the wing-tail configuration. (b) Lift curves for the airfoil, the wing-tail configuration and the individual contributions of the wing and the tail.
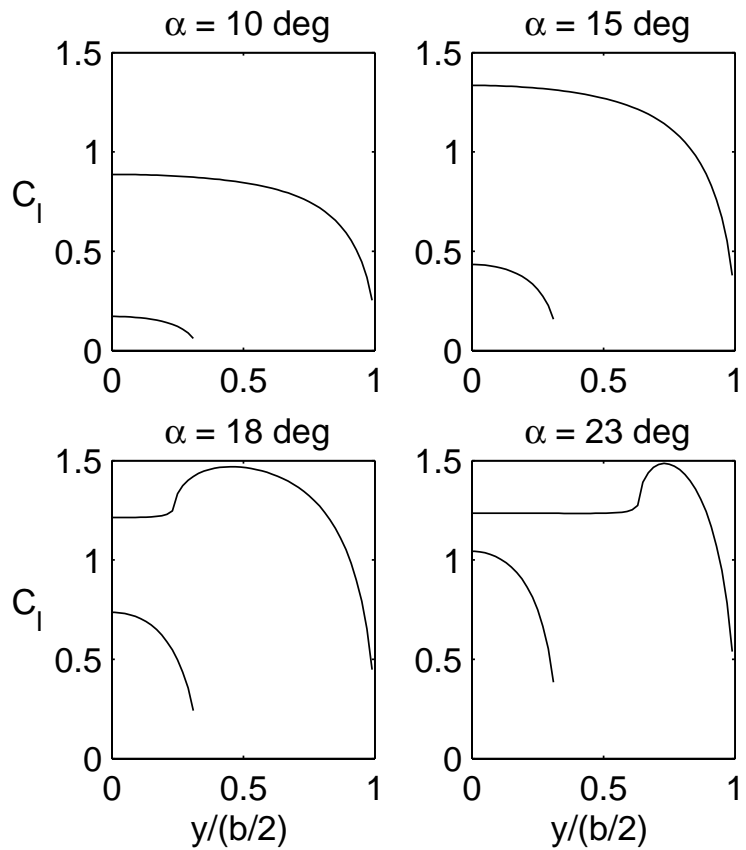

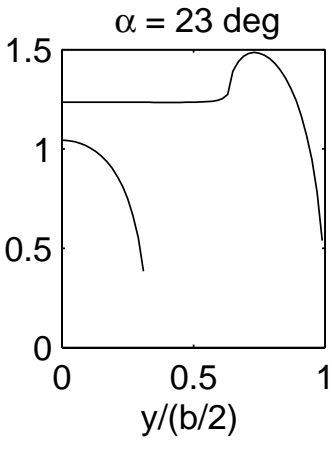

(a) Spanwise $C_{l}$ distributions

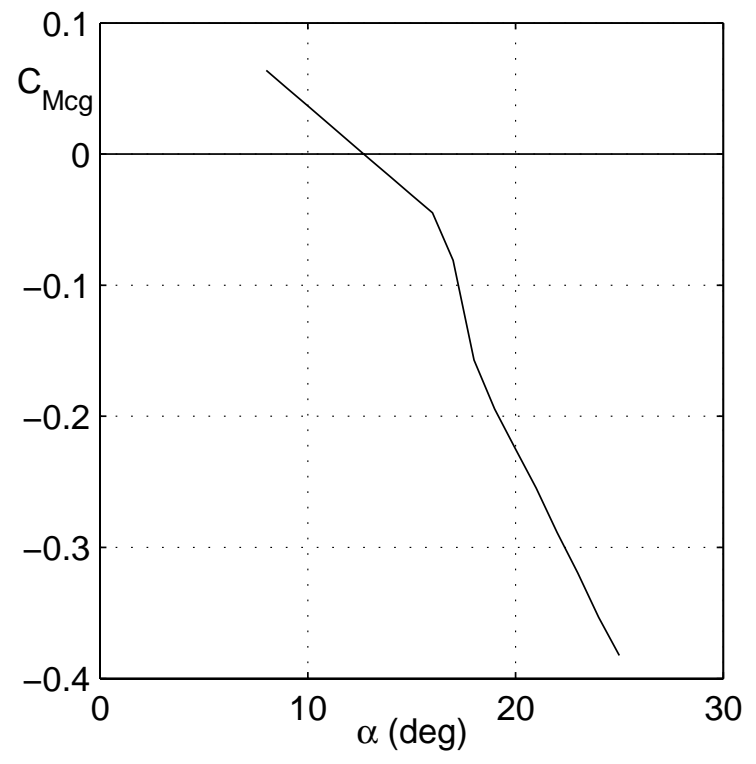

(b) Pitching-moment curve

Figure 6. (a) Spanwise $C_{l}$ distributions for the wing-tail configuration. (b) Pitching-moment curve for the wing-tail configuration. 


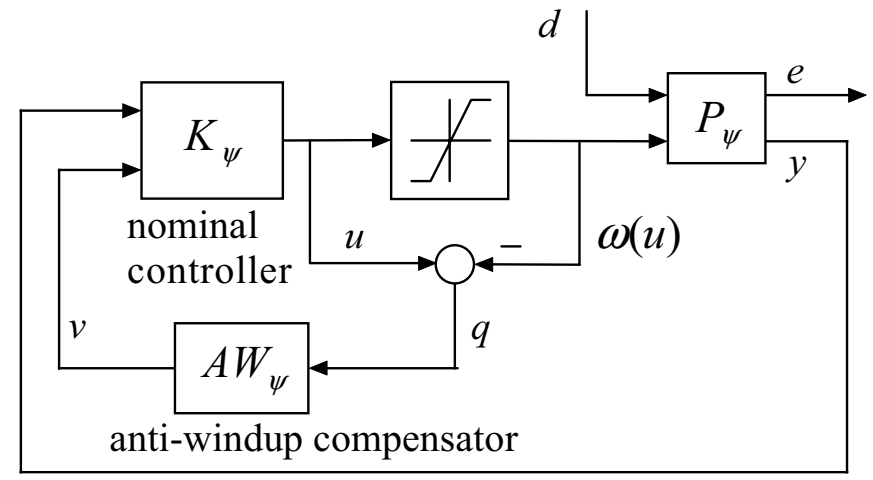

(a) LPV antiwindup control structure

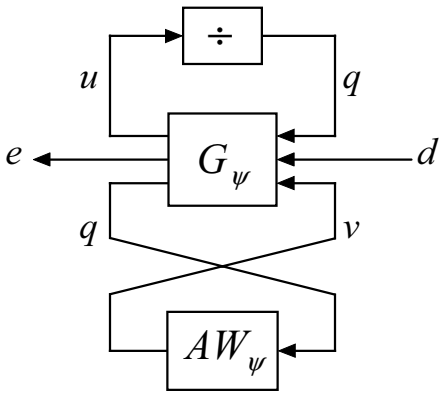

(b) Equivalent transformation

Figure 7. Nonlinear saturation control diagram.

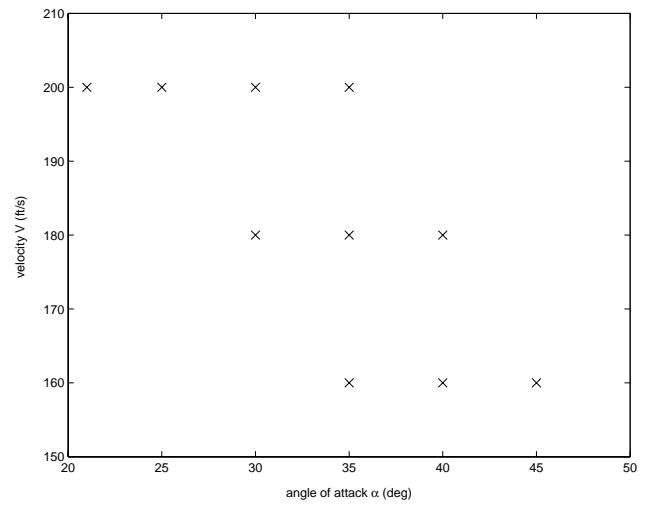

Figure 8. Flight trim points. 


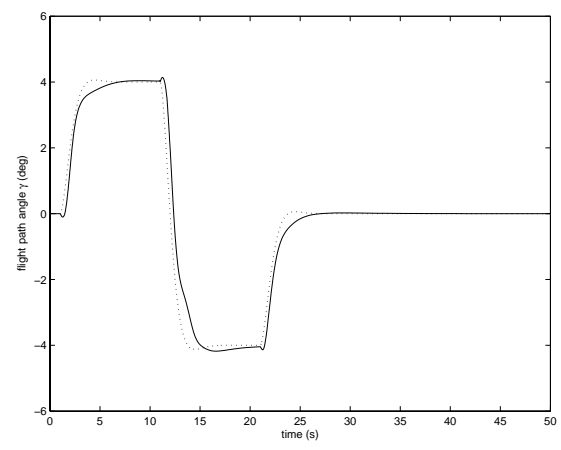

(a) Flight-path angle

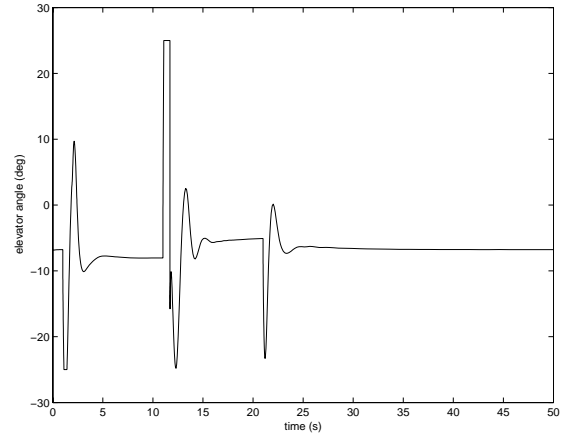

(c) Elevator angle

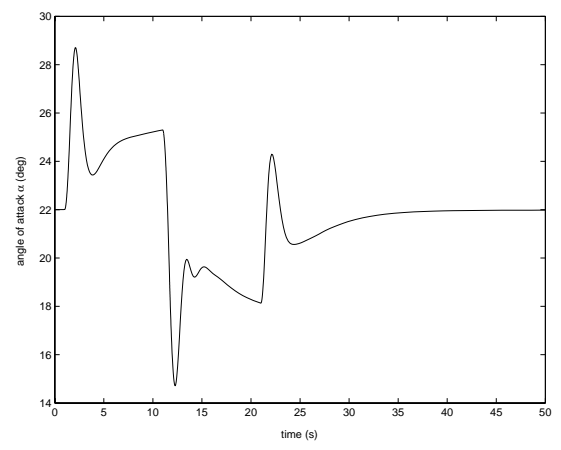

(b) Angle-of-attack

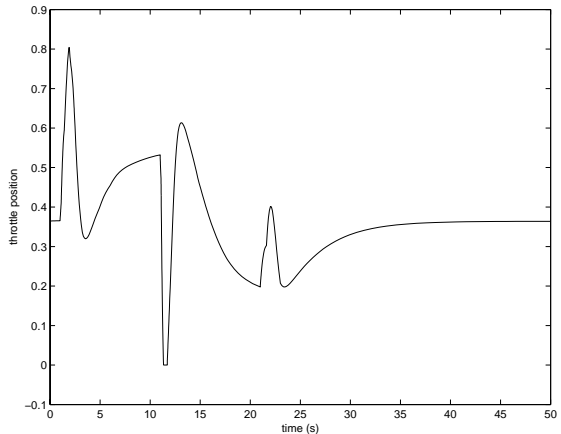

(d) Throttle position

Figure 9. Nonlinear doublet response with LPV antiwindup compensator: command signal (dot), actual response (solid).

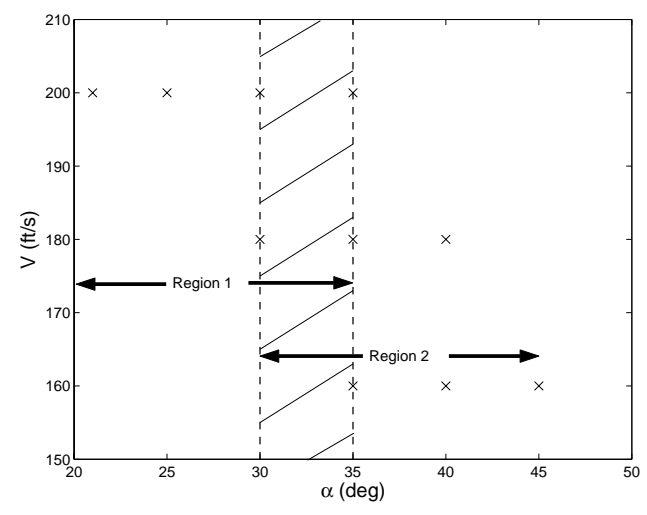

(a) Hysteresis switching

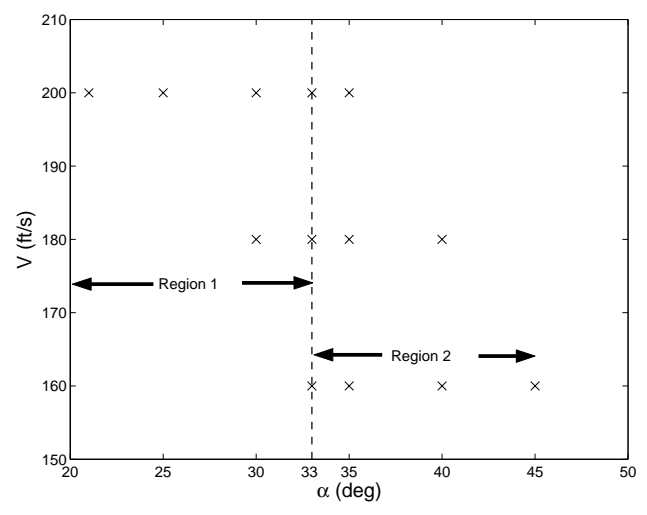

(b) Switching with average dwell time

Figure 10. Flight conditions and partitioned flight envelope for two switching logics. 


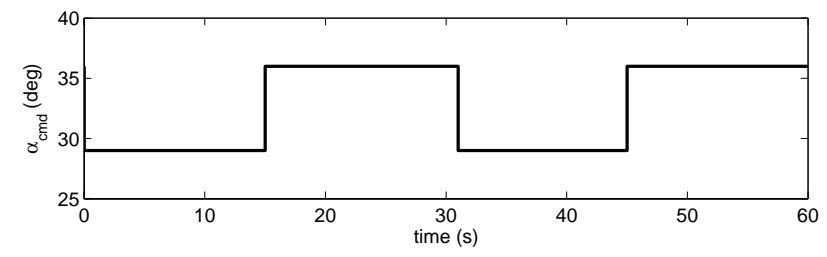

(a) Command input 1

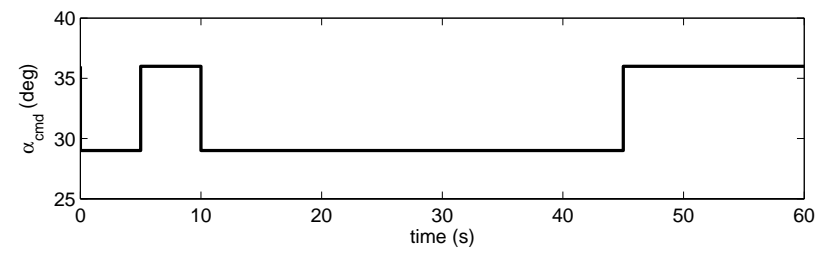

(b) Command input 2

Figure 11. Command inputs for switching LPV control simulation.

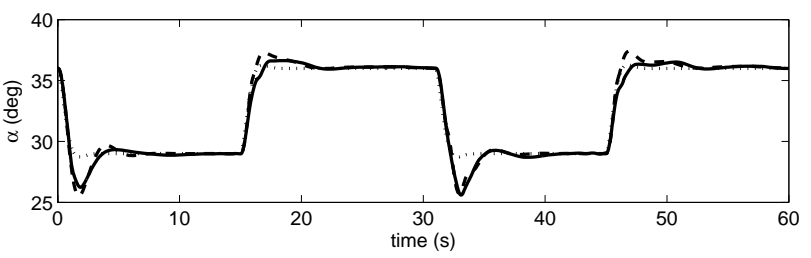

(a) Angle of attack

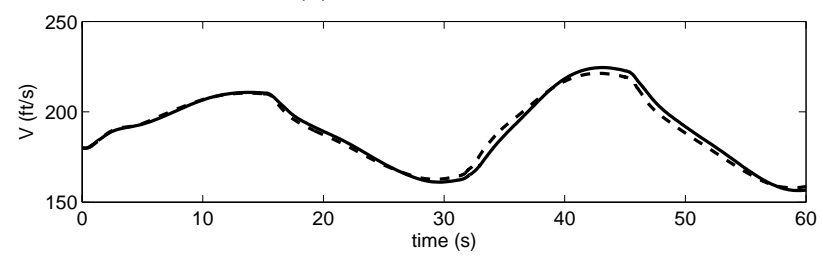

(c) Velocity

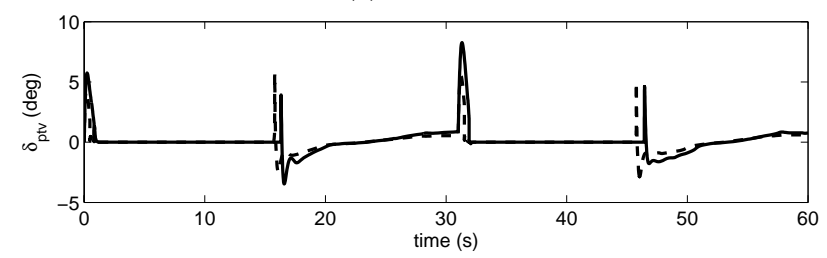

(e) Thrust vectoring nozzle deflection

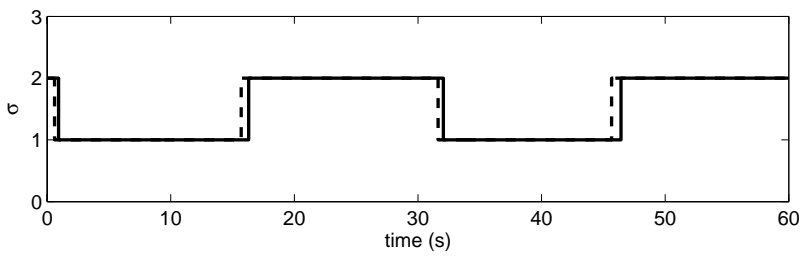

(b) Switching signal

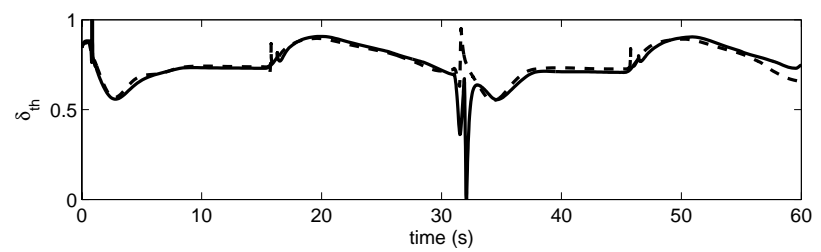

(d) Throttle position

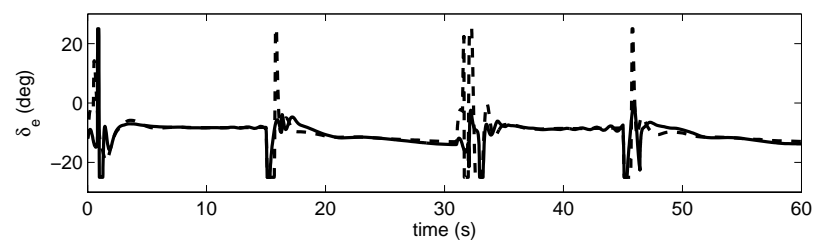

(f) Elevator angle

Figure 12. Nonlinear simulation of switching LPV control for command input 1: command (dot), hysteresis switching response (solid), dwell-time switching response (dash) 


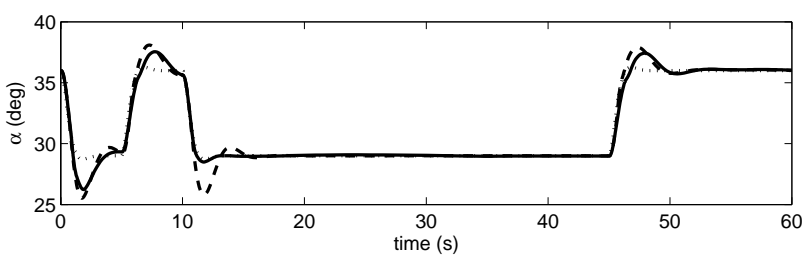

(a) Angle of attack

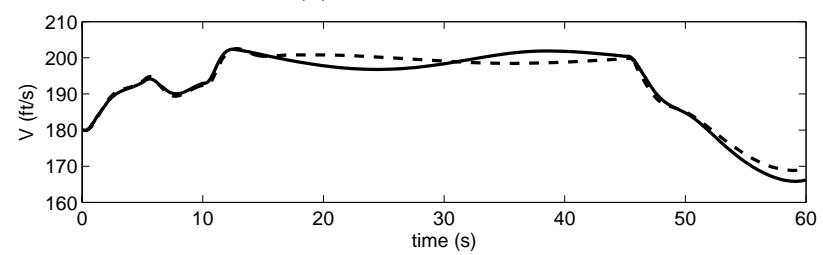

(c) Velocity

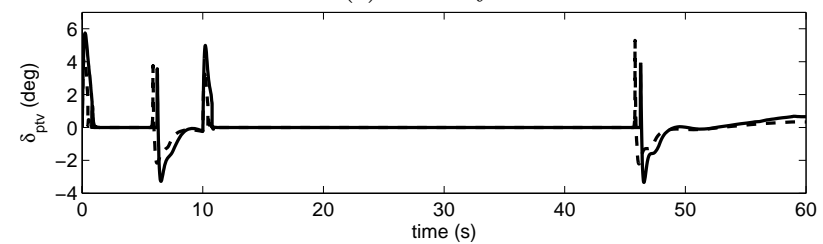

(e) Thrust vectoring nozzle deflection

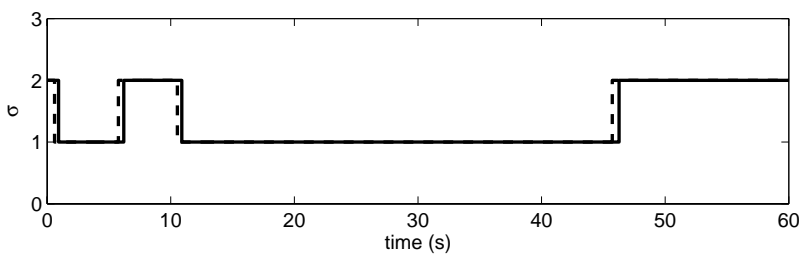

(b) Switching signal

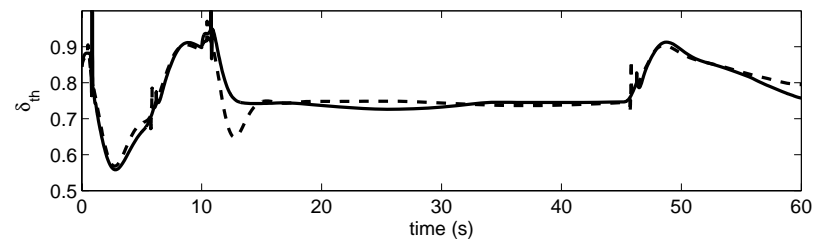

(d) Throttle position

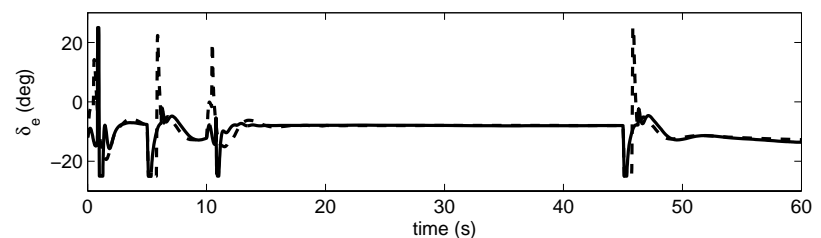

(f) Elevator angle

Figure 13. Nonlinear simulation of switching LPV control for command input 2: command (dot), hysteresis switching response (solid), dwell-time switching response (dash). 